\title{
PRODUCTIVIDAD TOTAL DE FACTORES DEL SECTOR CONSTRUCCION EN CHILE (1986-2015)
}

TOTAL FACTOR PRODUCTIVITY FOR THE CHILEAN CONSTRUCTION SECTOR (1986-2015)

\section{BYRON J. IDROVO-AGUIRRE}

Gerencia de Estudios Cámara Chilena de la Construcción

\section{VICTOR DANIEL SEREY}

Gerencia de Estudios Cámara Chilena de la Construcción

\begin{abstract}
In this paper, we estimate the Total Factor Productivity (TFP) for the Chilean Construction Sector, considering an annual period between 1986 and 2015. The contribution of the productivity to the sectorial growth is estimated residually, based on a Cobb-Douglas production function for the sectorial GDP and independent measures for capital and labor, corrected by quality and intensity of use. As a result, the sectorial TFP has exhibited a downward trend in the last five years, after remaining virtually flat since 1986 to 2011. The economic growth of the sector shows to be dominated by the accumulation of factors rather than by the efficiency with which they are used in the productive process. This could explain, partially, the vulnerability of sectorial activity to the economic cycle.
\end{abstract}

Keywords: Productivity, construction, Kalman filter, Chile.

JEL Classification: D24, E22, E23, E24.

\section{Resumen}

En el presente documento se estima la Productividad Total de Factores (PTF) para el Sector Construcción en Chile, considerando el período anual 1986-2015. La contribución de la productividad al crecimiento sectorial es estimada residualmente, a base de una función de producción Cobb- 
Douglas para el PIB de la construcción y medidas independientes de los factores primarios (capital y trabajo), ajustados por su calidad e intensidad de uso. Como resultado, la PTF muestra una tendencia decreciente en los últimos cinco años, luego de que permaneciera prácticamente plana en el período 1986-2011. Así, el crecimiento económico del sector mostraría estar más dominado por la acumulación de factores que por la eficiencia con que estos son utilizados en el proceso productivo. Esta situación podría explicar, en parte, la vulnerabilidad de la actividad sectorial a los avatares del ciclo económico.

Palabras clave: Productividad, construcción, filtro de Kalman, Chile.

Clasificación JEL: D24, E22, E23, E24.

\section{INTRODUCCION}

El modelo neoclásico de crecimiento económico, desarrollado por Solow (1957), define a la productividad total de factores (PTF) como aquella parte del Producto Interno Bruto (PIB) que se genera por la utilización eficiente de los factores primarios de producción (capital y trabajo). Bajo este enfoque, un aumento permanente de la productividad conduce a mayores y sostenidas tasas de crecimiento, más allá de los niveles explicados por el propio acervo del trabajo y stock de capital. En este sentido, se dice que un país o sector económico es productivo si es capaz de producir más con los mismos recursos o producir lo mismo con menos recursos.

En la versión más simple del modelo neoclásico, la PTF se obtiene de manera residual, es decir, la productividad es el resultado de restar las contribuciones tanto del trabajo como del capital al crecimiento real del PIB. Así, el remanente (o productividad) es "ese algo" relacionado con la "inspiración" de las firmas que promueven el crecimiento económico. En Chile, si bien existe un relativo consenso respecto de la metodología de cálculo de la PTF, las principales variantes de la literatura en esta materia provienen de los esfuerzos por obtener estimaciones más precisas de las variables trabajo y stock de capital $^{1}$. En efecto, bajo los supuestos de la teoría económica que respaldan el trabajo de Solow, la ganancia de ajustar apropiadamente por la calidad y la intensidad de uso a los factores básicos de producción se traduce en estimaciones menos sesgadas o más realistas de la productividad. A pesar de que, como en todo residuo, la PTF también incluye algo de la "medida de nuestra ignorancia", su utilidad sigue vigente

1 Variados son los trabajos que han orientado sus esfuerzos en depurar las medidas de los factores de producción, algunos de ellos son: De Gregorio (1997), Rojas et al. (1997), Roldos (1997), Coeymans (1999), De Gregorio y Lee (1999), Chumacero y Fuentes (2001), Bergoeing et al. (2002), Beyer y Vergara (2002), Contreras y García (2002), Gallego y Loayza (2002), Solimano y Soto (2003), Fuentes et al. (2004), Magendzo y Villena (2012). 
para explicar empíricamente el crecimiento económico de los países ${ }^{2}$. Por ejemplo, Fuentes et al. (2004), aprovechando la información del consumo de energía para capturar la intensidad de uso del capital ${ }^{3}$, logran obtener valores más depurados de la contribución de la productividad al crecimiento del PIB.

A nivel sectorial, este tema ha cobrado un renovado interés en Chile durante la última década ${ }^{4}$. No obstante, las estimaciones de PTF sectorial suelen ser de menor calidad que su par nacional, debido a que son pocos los sectores de la economía que cuentan con información estadística completa, tanto en términos de su confiabilidad como en relación con su extensión (información histórica). Por ejemplo, Vergara y Rivero (2006), Astorga (2011) y De la Huerta y García (2016) estiman la PTF sectorial sin ajustar por calidad e intensidad de uso a los factores trabajo y stock de capital. Entre sus resultados se observa que la productividad del sector construcción exhibió una tendencia decreciente en el período 1986-1999, para luego mantenerse relativamente plana en los siguientes años del horizonte de estimación. Por su parte, Betancor (2009) estima la PTF solo para el sector construcción, cuyo análisis considera los años de educación y horas trabajadas como instrumentos de ajuste por calidad e intensidad de uso de la mano de obra. Con relación al capital, su intensidad de uso fue ajustada por las desviaciones a la tendencia del índice de despachos de cemento $^{5}$. La PTF resultante es prácticamente plana durante el período 1986-2007, lo que sugiere un escaso protagonismo de la productividad en la dinámica del PIB de la construcción.

No obstante, este hallazgo debe ser interpretado con cierta cautela, ya que el componente residual del sector construcción tiene implícito varios efectos cruzados que pueden estar ocultando problemas de agregación. Por ejemplo, innovaciones a la política urbana y territorial que conlleven modificaciones y actualizaciones de normas de construcción, en el agregado, se pueden manifestar en progresos en la calidad del stock de capital. Al respecto, podemos mencionar las modificaciones a la norma de contructibilidad sísmica ${ }^{6}$ establecidas en los años 2010 y 2011, así como las nuevas exigencias técnicas dentro de la normativa del itemizado técnico del Programa de Fondo Solidario de Elección de Vivienda. Ambas normativas fueron establecidas por el Ministerio de Vivienda y Urbanismo (MINVU), lo que ha significado cambios a las especificaciones de tratamiento de suelos, materialidad, espacios, terminaciones y otros aspectos, tanto de construcciones en altura como en extensión para viviendas

Fuentes et al. (2004).

3 Esto como medida alternativa al ajuste tradicional a la Solow, basado en la razón entre la tasa de desempleo efectiva y la tasa de desempleo no aceleradora de la inflación (NAIRU por su siglas en inglés).

4 Basado en las primeras publicaciones que tratan el tema de la productividad total de los factores a nivel de sectores económicos.

5 El cálculo de la tendencia del indicador fue aproximada por el filtro de Hodrick y Prescott.

6 NCh 433 y NCh 2349. 
sociales $^{7}$. En efecto, estas medidas alteran la calidad de una fracción del stock de capital existente.

La presente investigación sigue de cerca el trabajo de Betancor (2009), en el sentido de que se analiza solo la productividad del Sector Construcción por tres motivos: (a) es altamente intensivo en el uso de mano de obra relativo tanto al capital como a otros sectores de la economía; (b) es un tema poco o nada explorado en Chile; y (c) los factores primarios de producción son ajustados por calidad e intensidad de uso por medio de instrumentos propios del sector, lo que limita la posibilidad de comparar los resultados con los obtenidos para el resto de los sectores de la economía. Asimismo, siguiendo el enfoque tradicional de Solow, la PTF sectorial y su contribución al crecimiento son estimados residualmente, a base de una función de producción CobbDouglas (con rendimientos constantes a escala) para el PIB de la construcción y las medidas independientes de capital y trabajo.

Aquí se amplían los trabajos previos en cuatro dimensiones: (i) se incrementa la ventana temporal de estudio, al usar datos anuales para el período 1986-2015; (ii) el stock de capital es ajustado por su intensidad de uso al ciclo económico empleando para ello las desviaciones a la tendencia de tres medidas alternativas del valor bruto de la construcción, estas son: el índice de fabricación de hormigón, el índice de despachos físicos industriales y el índice de fabricación de cemento ${ }^{8}$; (iii) se utiliza el filtro de Kalman como medida de tendencia alternativa al filtro de Hodrick y Prescott en la elaboración de medidas de ajuste de los factores; (iv) basado en investigaciones previas, se emplean múltiples coeficientes de participación del trabajo que, bajo el supuesto de rendimientos constantes a escala de la función de producción, permiten generar medidas alternativas de productividad de la construcción. En este punto, los resultados de Betancor (2009) son comparados con un intervalo al $95 \%$ de confianza en torno a la media de las múltiples estimaciones de PTF. Lo anterior es desarrollado con el propósito de responder a las siguientes dos interrogantes: ¿Son robustas las estimaciones anteriores a distintas y más recientes medidas de capital? y ¿En qué medida ha contribuido la PTF de la construcción al crecimiento del PIB del mismo sector?

El presente documento se organiza como sigue: luego de la introducción, la segunda sección describe la metodología para el cálculo de la PTF y las técnicas de ajuste aplicadas a los factores primarios de producción. En la sección 3 se presentan las medidas de stock de capital y trabajo, al mismo tiempo que se discute la importancia de ajustar por calidad e intensidad de uso de los factores. En la sección 4 se presentan las estimaciones de PTF para la construcción y la descomposición del crecimiento del PIB sectorial en sus tres fuentes primarias de crecimiento (contribución del

7 Por ejemplo, y para mayor detalle, ver Resolución № 6624, de octubre de 2016.

8 Los indicadores analíticos de fabricación de cemento y hormigón son publicados por el Instituto Nacional de Estadística (INE), mientras que el indicador de despachos físicos industriales de la construcción es elaborado por la Cámara Chilena de la Construcción (CChC). 
trabajo, del capital y de la productividad). Finalmente, en la sección 5 se entregan las conclusiones de este trabajo.

\section{METODOLOGIA}

El cálculo de la productividad total de factores y la descomposición de las fuentes del crecimiento del PIB de la construcción se basan en el modelo tradicional de Solow (1957). En este modelo se supone que el producto $(y)$ proviene de una función de producción continua y diferenciable $(F)$, cuyos argumentos son los factores primarios de producción: capital físico $(k)$ y trabajo $(l)^{9}$. Formalmente, se tiene:

$$
y=F(k, l), \quad \text { donde } F_{k}, F_{l}>0 \text { y } F_{k k}, F_{l l}<0
$$

Se supone además una firma representativa en una economía donde hay competencia perfecta, tanto en el mercado de bienes como en el mercado de factores. Bajo este enfoque, la decisión de producción óptima (aquella que maximiza el beneficio de la firma) ocurre cuando la productividad marginal del capital es igual a la tasa de interés del mercado $(r)$, al mismo tiempo que la productividad marginal del trabajo se equipara con el salario $(w)$. Formalmente, el problema que resuelve la firma es:

$$
\max _{k, l} \Pi \equiv p F(k, l)-w l-r k
$$

A partir de las condiciones necesarias de primer orden (c.n.p.o) aplicadas a la función de beneficios que enfrenta la firma (función objetivo), el nivel de producción óptimo se alcanza cuando las productividades marginales de los factores se igualan a sus respectivos costos marginales:

$$
p F_{k}=r, \quad p F_{l}=w
$$

donde $p$ es el valor unitario del bien producido. Tomando la derivada total a la función de producción $F$, se tiene:

$$
\frac{d y}{y}=F_{k}\left(\frac{k}{y}\right) \frac{d k}{k}+F_{l}\left(\frac{l}{y}\right) \frac{d l}{l}
$$

9 En el caso particular de una función de producción de Cobb-Douglas con rendimientos constantes a escala, tiene la siguiente representación explícita: $F(k, l)=A k^{\alpha} l^{1-\alpha}$, Donde $A$ captura el componente de productividad total de los factores y el parámetro $\alpha$ es la incidencia de la acumulación de trabajo en el producto. Debido al supuesto de rendimientos constantes a escala, entonces $(1-\alpha)$ representa la participación del capital en el producto. 
Reemplazando las (c.n.p.o) en la expresión anterior, y llamando a las variables $\frac{d y}{y}, \frac{d k}{k}$ y $\frac{d l}{l}$ como tasas de crecimiento $\Delta \% y, \Delta \% k$ y $\Delta \% l$, respectivamente, se obtiene que el crecimiento del PIB $(\Delta \% y)$ queda definido por:

$$
\Delta \% y=\left(\frac{r k}{p y}\right) \Delta \% k+\left(\frac{w l}{p y}\right) \Delta \% l
$$

De la expresión anterior se desprende que el crecimiento del producto se define como un promedio ponderado del crecimiento de los factores básicos de producción. Los ponderadores corresponden entonces a las participaciones valoradas de cada insumo en el producto total. Bajo el supuesto de competencia perfecta, la suma de ambos ponderadores es igual a la unidad (similar al supuesto de rendimientos constantes a escala en la función de producción), por lo que el pago de los factores agota el producto o, en otras palabras, todo el ingreso generado por las firmas se destina al pago por el uso de los factores.

Suponga ahora que el producto $(y)$ se expande a una tasa mayor a la predicha por la acumulación de factores. Entonces ese diferencial se atribuye al progreso tecnológico o crecimiento de la productividad $\Delta \% A$ de manera que:

$$
\Delta \% A=\Delta \% y-(1-\alpha) \Delta \% k-\alpha \Delta \% l
$$

donde $\alpha$ es una reparametrización de la participación del trabajo en el PIB $\left(\frac{w l}{p y}\right)$. Como se puede observar, el crecimiento de la productividad queda entonces definido como un residuo, el que es llamado residuo de Solow en la teoría neoclásica. Como todo residuo, este está sujeto a problemas de especificación en su forma funcional, omisión de variables relevantes y problemas de medición de los factores primarios de producción. Sin embargo, pese a todas sus limitaciones, el cálculo de la PTF, bajo este enfoque tradicional, sigue siendo un instrumento válido y relevante para analizar el crecimiento económico de los países.

\subsection{La medida de productividad con ajuste por calidad e intensidad de uso de los factores}

Si consideramos los factores ajustados por su calidad e intensidad de uso en cada etapa del ciclo económico, entonces tenemos una nueva versión de la función de producción anterior:

$$
y=F(k \phi, l(h)(e)), \quad F_{k \phi}, F_{l(h)(e)}>0
$$


donde $k \phi$ representa el capital físico ajustado por intensidad de uso $\phi$. Asimismo, el trabajo $l$ es ajustado por las horas trabajadas $(h)$-que captura la intensidad de uso del factor-y los años de educación $(e)$-que intenta medir la calidad del trabajo-.

Respecto del factor capital, se emplean en este documento nueve medidas alternativas (la variable tradicional y ocho medidas ajustadas por el grado de utilización del factor). A continuación se destacan las características más importantes de estas:

- La variable tradicional de stock de capital es obtenida directamente de las estadísticas de Cuentas Nacionales (CCNN) del Banco Central de Chile (BCCh). Esta versión original del capital es comúnmente usada cuando no se tiene información respecto de la calidad o intensidad de uso del factor.

- La variable de stock de capital ajustado por su grado de utilización sigue el procedimiento usado por el Ministerio de Hacienda para el cálculo del PIB potencial. En este caso, el instrumento de uso del capital es el ratio (desviación) entre la tasa de ocupación del mercado laboral y la tasa de ocupación tendencial (conocido como "ajuste a la Solow"): $k \phi \equiv k\left(\frac{1-\mu}{1-\mu^{T}}\right)$, siendo $\mu$ y $\mu^{T}$ las tasas de desempleo efectivo y desempleo natural, respectivamente.

- La variable de stock de capital ajustado por su grado de utilización corresponde a las desviaciones a la tendencia de las series de fabricación de cemento, hormigón y el índice de despachos físicos industriales. Adicionalmente se obtuvieron dos medidas de tendencia: la tradicional de Hodrick y Prescott y la no tradicional basada en el filtro de Kalman. En este sentido, se lograron siete medidas alternativas de capital ajustado por utilización: $k \phi \equiv k\left(\frac{\tau}{\tau^{T}}\right)$, siendo $\tau$ la medida de la serie de ajuste efectiva y $\tau^{T}$ su tendencia de largo plazo.

Particularmente, la estimación de la tendencia por el método del filtro de Kalman está basada en la siguiente especificación de un modelo de estado-espacio:

$$
\begin{gathered}
\tau_{T}=\tau_{t}^{C}+\tau_{t}^{T} \\
\tau_{t}^{T}=\varrho_{t}+\tau_{t-1}^{T}+\varepsilon_{\tau} \\
\varrho_{t}=\varrho_{t-1}+\varepsilon_{\varrho} \\
\tau_{t}^{C}=\varphi_{1} \tau_{t-1}^{C}+\varphi_{2} \tau_{t-2}^{C}+\varepsilon_{\varphi}
\end{gathered}
$$

donde las series de ajuste (cemento, hormigón y despachos físicos) se descomponen en dos términos: uno cíclico $\tau_{t}^{C}$ y otro tendencial $\tau_{t}^{T}$, junto con sus respectivas 
dinámicas internas. La ventaja del filtro de Kalman respecto del filtro de Hodrick y Prescott es que la tendencia resulta de una dinámica explícita y no únicamente de un parámetro de penalización o suavización de la serie de tiempo.

Por otra parte, se utilizan tres medidas alternativas de ajuste del trabajo por intensidad de uso y calidad del insumo. Ello, sumado a la medida tradicional de empleo (número de ocupados sin ajustar por utilización y calidad), conforman cuatro variables alternativas de ocupados del sector construcción:

- La variable tradicional de empleo $(l)$ es obtenida directamente de las estadísticas INE. Esta versión original del empleo, medida como el número de ocupados del sector, es comúnmente usada cuando no se tiene información respecto de la calidad o intensidad de uso del factor.

- La variable empleo ajustada por los años de educación (e). Esta variable intenta medir la calidad del empleo y se define como el producto $l^{*} e$.

- La variable ocupados ajustada por las horas efectivamente trabajadas $(h)$, que permite aproximar la intensidad de uso de la mano de obra. El cálculo se obtiene de multiplicar $l * h$.

- Finalmente, el ajuste del trabajo por calidad como por su intensidad de uso se define como el producto $l^{*} e^{*} h$. Como se puede notar, esta variable combina las dos medidas de ajuste anteriores ${ }^{10}$.

Finalmente, las ecuaciones para estimar la tasa de crecimiento de la productividad total de factores ajustada son de la forma:

$$
\Delta \% \tilde{A}=\Delta \% y-(1-\alpha) \Delta \%(k \phi)-\alpha \Delta \%(l(e)(h))
$$

Como se puede apreciar, solo se requiere de un parámetro $\alpha$ para obtener las medidas de productividad a partir de las series observadas de los factores capital y trabajo. A partir de la información de Cuentas Nacionales del BCCh, los trabajos de Betancor (2009) y De la Huerta y García (2016), se obtuvieron las participaciones del trabajo sectorial en el PIB de la construcción para los años en estudio, así como su promedio simple para el período 1986-2015. Es importante notar que esta medida podría estar sesgada negativamente, debido a la metodología utilizada por el INE para definir los ocupados del sector construcción. Una forma de corregir este sesgo, aunque parcialmente, es aplicando la técnica de Vergara y Rivero (2006), donde las participaciones del trabajo a nivel sectorial son reescaladas según los desvíos de las participaciones agregadas entre CCNN y la información entregada por Hacienda para el cálculo del PIB potencial. Adicionalmente se realizó un análisis de sensibilidad

10 Las series de años de educación y las horas efectivamente trabajadas provienen de las bases de microdatos, Universidad de Chile. 
variando el parámetro $\alpha$ y los resultados no exhiben cambios disímiles en términos tendenciales.

\section{MEDIDAS DE CAPITAL, TRABAJO Y PARAMETROS DE PARTICIPACION DE LOS FACTORES BASICOS DE PRODUCCION EN EL PIB DE LA CONSTRUCCION}

A continuación se presentan las medidas alternativas tanto de los factores básicos de producción como de la participación de la mano de obra en el PIB de la construcción. Para el stock de capital sectorial se considera, además de su medición original de Cuentas Nacionales del BCCh, las correcciones por su intensidad de uso en las distintas etapas del ciclo económico. De igual forma, la mano de obra considera en su evolución los cambios en la calidad y la tasa de utilización, ambos aproximados por los años de educación y las horas trabajadas. En tanto, las mediciones del parámetro de participación de los factores en el PIB están basadas en los trabajos de Betancor (2009), De la Huerta y García (2016), entre otras fuentes complementarias, como los boletines anuales de Cuentas Nacionales del Banco Central y las bases de datos del Ministerio de Hacienda para el cálculo del PIB potencial a nivel nacional. Esto con el propósito de generar medidas más precisas de la productividad total de factores.

\subsection{Medidas de capital}

La medida básica de stock de capital para el sector construcción es el capital invertido en obras de edificación y obras civiles, cuya fuente es Cuentas Nacionales del BCCh. Al respecto, es factible mencionar que esta variable de stock de capital no estaba disponible de manera oficial durante el desarrollo de la mayoría de trabajos previos. Por lo que Vergara y Rivero (2006), Betancor (2009) y Astorga (2011) utilizaron sus propias estimaciones de capital físico de la construcción, basadas en el método de inventarios perpetuos. En este sentido, la presente investigación también contribuye a evaluar la robustez de las estimaciones anteriores de productividad.

En principio, toda medida básica de capital representa el stock disponible en la economía y no el que efectivamente están utilizando las firmas en sus procesos de producción para cada momento del tiempo. Por lo que el stock de capital, en su versión original (sin ajustar), no refleja las variaciones en su tasa de utilización durante las distintas fases del ciclo económico. Por ejemplo, el capital físico existente podría no ser empleado en su total capacidad durante períodos recesivos. Así, para efectos del presente estudio lo que interesa es el stock de capital efectivamente utilizado. De no ajustar el capital por su intensidad de uso, entonces todas las medidas residuales de la PTF reflejarán también la subutilización del factor, arrojando un resultado sesgado de la verdadera productividad. 
Con el objetivo de realizar dicho ajuste, se proponen ocho medidas alternativas de stock de capital ajustado. Además, considerando el stock de capital básico (sin ajustar) de Cuentas Nacionales, suman nueve medidas alternativas de stock de capital para el cálculo de la PTF. Como primer instrumento de ajuste se utiliza la tasa de ocupación sectorial. Esta medida supone que el capital y el trabajo tienen una tasa de uso similar, es decir, una variación en la capacidad de uso del capital va de la mano con los cambios en la demanda por trabajo. Se asume entonces la existencia de una alta complementariedad entre los factores trabajo y capital. Pese a las limitaciones del supuesto, esta medida de ajuste es empleada por el Ministerio de Hacienda para el cálculo del PIB potencial.

No obstante, como medidas alternativas de ajuste por intensidad de uso del capital, aquí se utilizan la información de fabricación de cemento, fabricación de hormigón y el índice de despachos físicos industriales para la construcción ${ }^{11}$. Así, los indicadores proxies del grado de utilización se construyen como el ratio entre la serie efectiva y su medida de tendencia de largo plazo. Las tendencias son obtenidas empleando el filtro de Hodrick y Prescott y el filtro de Kalman, respectivamente. En conclusión, al multiplicar el stock de capital básico por estas desviaciones a la tendencia, se obtienen las nuevas series de capital ajustadas por su grado de utilización en cada momento del tiempo.

La Figura 1 muestra la evolución de las nueve series alternativas de stock de capital de la construcción previamente descritas, y donde en el panel superior se comparan las medidas de tendencia obtenidas mediante un filtro Hodrick y Prescott, mientras que el panel inferior son las series de capital ajustadas con un filtro de Kalman. Lo primero que se desprende de ambos gráficos es que las medidas de capital poseen en su mayoría una dinámica similar en términos de sus tendencias, aunque difieren en sus medidas de volatilidad ${ }^{12}$. Básicamente, el stock de capital ajustado por su intensidad de uso, mediante la tasa de ocupación y los tres instrumentos relacionados con la actividad del sector (cemento, hormigón y despachos industriales), muestran una mayor volatilidad relativa a la observada en la evolución del capital sin ajustar. Esto último significa que el stock de capital ajustado da cuenta de que el grado de utilización del factor no es una constante a lo largo del tiempo. Por otra parte, al comparar ambos paneles (superior e inferior) se puede apreciar que los ajustes de capital que utilizaron

11 Los indicadores analíticos de fabricación de cemento y hormigón son provistos por el INE, mientras que los despachos físicos industriales corresponden a las estadísticas de la CChC.

12 Las medidas de correlación simple entre los distintos indicadores de stock de capital oscilan entre 0,95 y 0,99 . Por ejemplo, la correlación más baja $(0,95)$ ocurre entre el stock de capital corregido por las desviaciones de tendencia de la fabricación de hormigón (usando el filtro de Hodrick y Prescott) y el stock de capital corregido por las desviaciones de tendencia del índice de despachos físicos industriales (usando el filtro de Hodrick y Prescott). Mientras que la correlación más alta $(0,99)$ es reportada por las variables stock de capital básico y el corregido por la tasa de desempleo. En términos de tasas de crecimiento anual, las medidas de correlación, en la mayoría de los casos, superan la medida de 0,7 con un nivel de significancia de $1 \%$. 


\section{FIGURA 1}

STOCK DE CAPITAL DE LA CONSTRUCCION SIN AJUSTAR Y AJUSTADO POR INTENSIDAD DE USO 1986-2015 (INDICE 1986 = 100) CON FILTRO DE HODRICK Y PRESCOTT (PANEL

SUPERIOR) Y FILTRO DE KALMAN (PANEL INFERIOR)
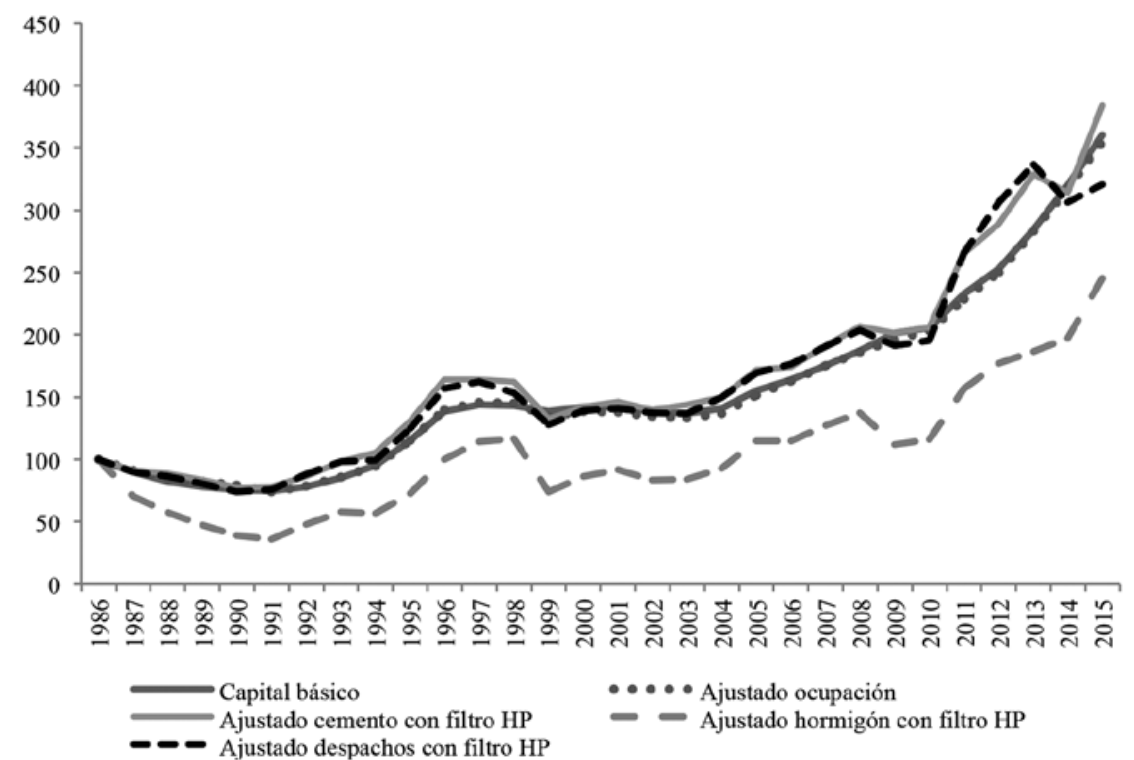

$\bullet \bullet$ Ajustado ocupación

- Ajustado hormigón con filtro HP

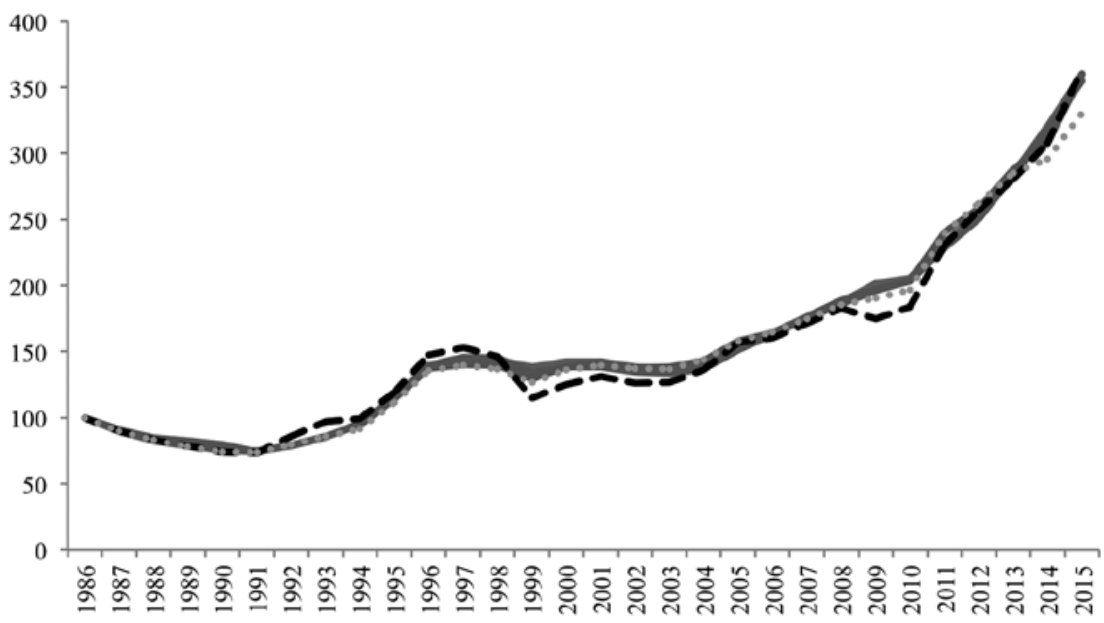

Capital básico

Ajustado cemento con filtro Kalman .... Ajustado despachos con filtro Kalman
Ajustado ocupación

- Ajustado hormigón con filtro Kalman 
como medida de tendencia el filtro de Kalman, reportan resultados más estables o robustos respecto de aquellos basados en el filtro de Hodrick y Prescott. En general, los ajustes de capital por la fabricación de hormigón, utilizando el filtro de Hodrick y Prescott como medida de tendencia, resultaron ser los más volátiles.

\subsection{Medidas de trabajo}

La medida básica de trabajo utilizada en este apartado es el número de ocupados del sector construcción, provistos por el INE durante el período 1986-2015. Las series correspondientes a las encuestas ENE (Encuesta Nacional de Empleo) y NENE (Nueva Encuesta Nacional de Empleo) fueron empalmadas mediante interpolación lineal ${ }^{13}$. El primer ajuste realizado a esta variable es para controlar por la intensidad de uso del factor, y para ello se consideró el promedio anual de las horas efectivamente trabajadas. La fuente de esta información es la Encuesta de Ocupados y Desocupados del Centro de Microdatos del Departamento de Economía de la Universidad de Chile, ya que se consulta la rama de actividad y las horas semanales trabajadas de ocupados de hogares del Gran Santiago. Luego, basado en la misma fuente de información, se considera un ajuste del trabajo por calidad, donde los años de educación son comúnmente utilizados como medida proxy de la calidad del empleo.

Al respecto es posible mencionar que no ajustar el trabajo por su grado de utilización y calidad podría sesgar la medición de la PTF. Por ejemplo, podría darse el caso en el que la PTF aumenta debido a una mejora en la calidad del empleo y no necesariamente a un aumento de la productividad. Por ello, elaboramos cuatro medidas alternativas de trabajo que son: (i) sin ajustar (medida tradicional), (ii) ajustado por educación (medida corregida por la calidad de la mano de obra), (iii) ajustado por educación y horas trabajadas (medida corregida por calidad e intensidad de uso del factor), y (iv) ajustado por horas trabajadas (medida corregida por el grado de utilización del trabajo).

La Figura 2 muestra la evolución de las series de empleo de la construcción, horas trabajadas (panel superior) y años de educación (panel inferior). De las figuras se desprende que las series de los ocupados de la construcción (medida en miles de trabajadores) y los años de escolaridad comparten tendencia positiva y sus movimientos cíclicos son relativamente similares (panel inferior). Por otro lado, las horas trabajadas en el sector de la construcción presentan una tendencia decreciente, es decir, se observa una disminución de las horas de mano de obra destinadas a la construcción durante el período anual de 1986-2015 (panel superior). Al respecto, los paneles $a$ y $b$ de la Tabla 1 muestran las

13 La base de empleo de microdatos (expresada en variación anual) y la base del INE (tomando la variación anual de la serie de empleo de la encuesta ENE) arrojan una correlación de 0,71. Esta medida es similar a la observada entre la base de microdatos y la serie Back-splicing de la CChC, creada para dar continuidad histórica a la nueva serie de empleo (NENE). Así, el ratio entre ambos valores de correlación es 0,99. De esta forma, se prefirió trabajar con la serie empalmada de empleo, por poseer mayor información histórica. 


\section{FIGURA 2}

EMPLEO, HORAS TRABAJADAS, AÑOS DE ESCOLARIDAD PARA EL SECTOR CONSTRUCCION EN CHILE 1986-2015
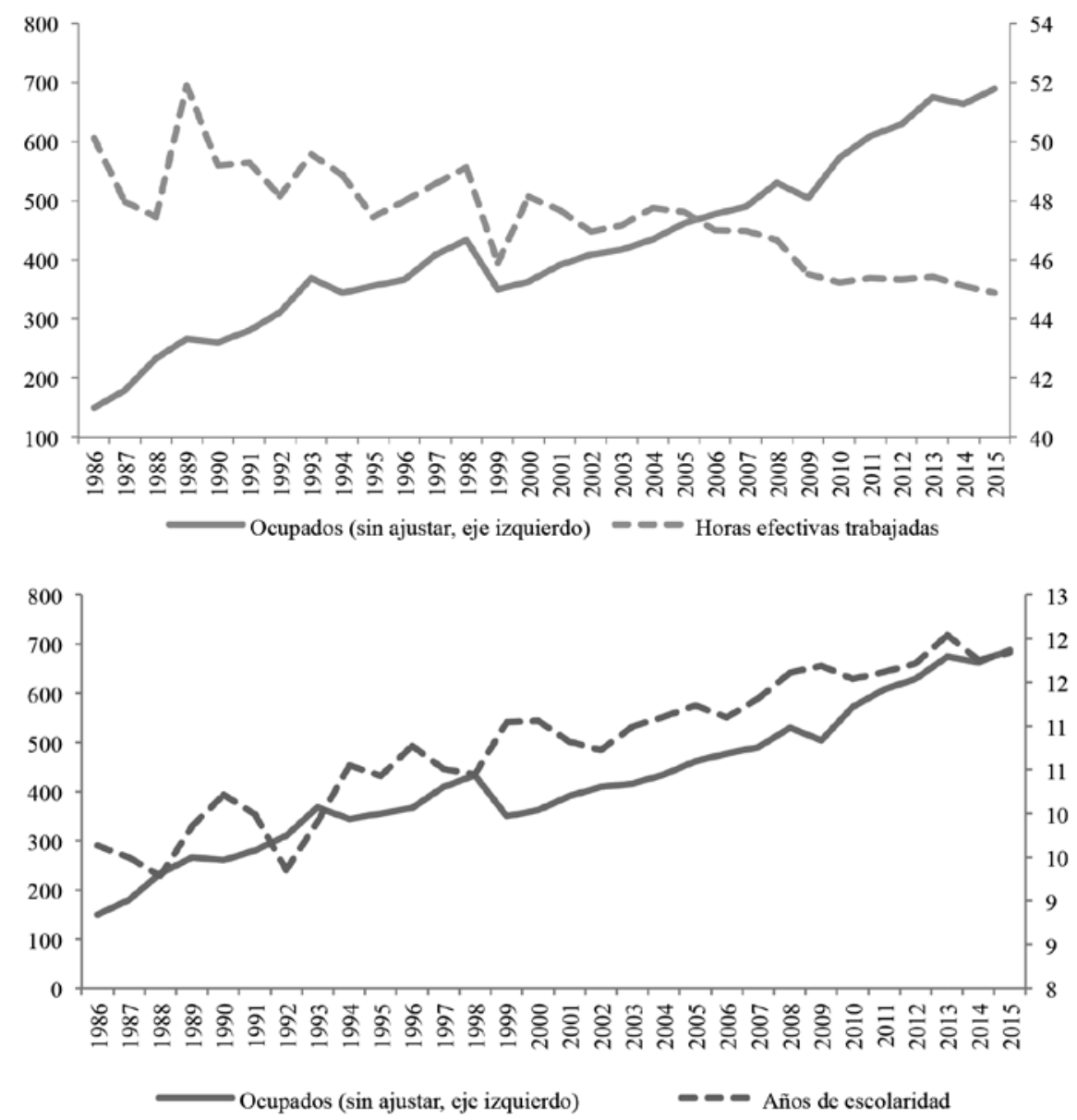

medidas de correlación simple entre las cuatro variables alternativas de empleo (sin ajustar y ajustadas por calidad y uso). El panel $a$ presenta las correlaciones en niveles, es decir, sin controlar por el efecto tendencia de las series, por lo que predomina la alta correlación de las tendencias (en torno a 0,99). De igual forma, el panel $b$, tomando en cuenta el efecto tendencia de las series de empleo, muestra una elevada correlación de las tasas de crecimiento anual, cercanas a 0,9 . 


\section{TABLA 1}

COEFICIENTES DE CORRELACION ENTRE DISTINTAS MEDIDAS DE TRABAJO, PARA LAS VARIABLES EN NIVEL (PANEL A) Y VARIABLES EN TASAS DE CRECIMIENTO (PANEL B)

(a) Variables en niveles

(Sin tomar en cuenta el efecto tendencia)

\begin{tabular}{|l|r|r|r|r|}
\cline { 2 - 5 } \multicolumn{1}{c|}{} & \multicolumn{4}{c|}{ Empleo } \\
\cline { 2 - 5 } \multicolumn{1}{c|}{ SA } & AHE & AE & \\
\hline SA & 1 & & & \\
AHE & 0,997 & 1 & 1 & 1 \\
AE & 0,9971 & 0,9984 & 0,9914 & 1 \\
AH & 0,9975 & 0,996 & \\
\hline
\end{tabular}

(b) Variables en tasas de crecimiento)

(tomando en cuenta el efecto tendencia)

\begin{tabular}{|l|r|r|r|r|}
\cline { 2 - 5 } \multicolumn{1}{c|}{} & \multicolumn{4}{c|}{ Empleo } \\
\cline { 2 - 5 } \multicolumn{1}{c|}{} & SA & AHE & AE & AH \\
\hline SA & 1 & & & \\
AHE & 0,8625 & 1 & 1 & 1 \\
AE & 0,9336 & 0,9623 & 0,9423 & 1 \\
AH & 0,9573 & 0,9494 & \\
\hline
\end{tabular}

SA: Empleo sin ajustar.

AHE: Empleo ajustado por horas trabajadas y educación (ajustado por calidad).

AE: Empleo ajustado por años de educación.

AH: Empleo ajustado por horas trabajadas.

\subsection{Medidas de contribución del trabajo al PIB de la construcción}

En esta sección se discuten los valores que toman los parámetros de participación de los factores básicos de producción en el PIB de la construcción. Así, considerando el supuesto de rendimientos constantes a escala en la forma funcional de Cobb-Douglas, es suficiente mostrar la tasa de participación de uno de los dos factores primarios de producción, ya que la suma de sus contribuciones al PIB sectorial es igual a la unidad. A base de la literatura existente, pareciera ser la norma enfocarse en la tasa de participación del trabajo, presuntamente por el motivo de ser una variable con menor error de medición respecto del stock de capital. En efecto, y siendo consistente con la literatura, el análisis de este apartado se centra en las medidas alternativas del parámetro de contribución del trabajo en la producción.

Con el propósito de realizar un exhaustivo análisis de sensibilidad de la medida residual de la PTF sectorial a distintas tasas de participación del trabajo en el PIB de 
la construcción, en esta sección se exponen 18 valores alternativos para este parámetro (que en la sección 2 de metodología fue llamado coeficiente $\alpha$ ). Los valores de este coeficiente provienen de los parámetros usados tanto por Betancor (2009) como por De la Huerta y García (2016), y de otras fuentes complementarias, como los boletines anuales de Cuentas Nacionales del BCCh y las bases del Ministerio de Hacienda para el cálculo del PIB potencial. Al respecto, y como fue mencionado previamente, la tasa de participación del trabajo sectorial obtenida desde la información de CCNN podría estar sesgada (hacia abajo), debido a la metodología utilizada por el INE para definir los ocupados del sector construcción. Una forma de corregir este sesgo, aunque parcialmente, es aplicando la técnica de Vergara y Rivero (2006) ${ }^{14}$.

En otras palabras, la corrección del parámetro de participación de la mano de obra en el PIB sectorial, obtenido de CCNN, consiste en los siguientes pasos: (i) a partir de la estimación agregada de PTF y las variables macroeconómicas de PIB, ocupados y stock de capital, publicadas anualmente por el Ministerio de Hacienda, se derivan los parámetros de participación del trabajo a nivel nacional. Esto está basado en el supuesto metodológico de que el producto puede ser representado por una función de producción de Cobb-Douglas con rendimientos constantes a escala; (ii) se obtienen las participaciones agregadas del factor trabajo a base de las estadísticas de CCNN del BCCh; (iii) se calculan las desviaciones de las medidas de participación del trabajo de Hacienda respecto de la obtenida a partir de CCNN; (iv) finalmente, las desviaciones calculadas en el paso anterior sirven de factor de ajuste de la tasa de participación de la mano de obra $(\alpha)$ en el sector construcción, obtenido de las estadísticas de CCNN del BCCh.

La Figura 3 muestra los valores alternativos del parámetro de participación del trabajo en el PIB de la construcción. Los parámetros variables son los calculados a partir de los boletines anuales de CCNN, donde se aplicó la técnica de ajuste previamente mencionada de Vergara y Rivero (2006). De la Figura 3 se desprende que, para la mayoría de las medidas alternativas de participación, se observa un aumento de la participación de la mano de obra para el período postcrisis asiática (1999-2006), situación que en promedio se ha mantenido en la última década, lo que, en parte, refleja el hecho de que el sector construcción sea uno de los sectores económicos altamente intensivos en el uso del trabajo. No obstante, a partir de los años 2012-2013 se observa un descenso de esta medida de participación, situación que podría acotar el crecimiento de largo plazo si no se destinan mayores esfuerzos a mejorar la productividad.

14 Donde las participaciones del trabajo a nivel sectorial son reescaladas según los desvíos de las participaciones agregadas entre las estadísticas de Cuentas Nacionales y las que se deducen de la información entregada por Hacienda para el cálculo del PIB potencial. 


\section{FIGURA 3}

MEDIDAS ALTERNATIVAS DE PARTICIPACION DEL TRABAJO EN EL PIB 1986-2015

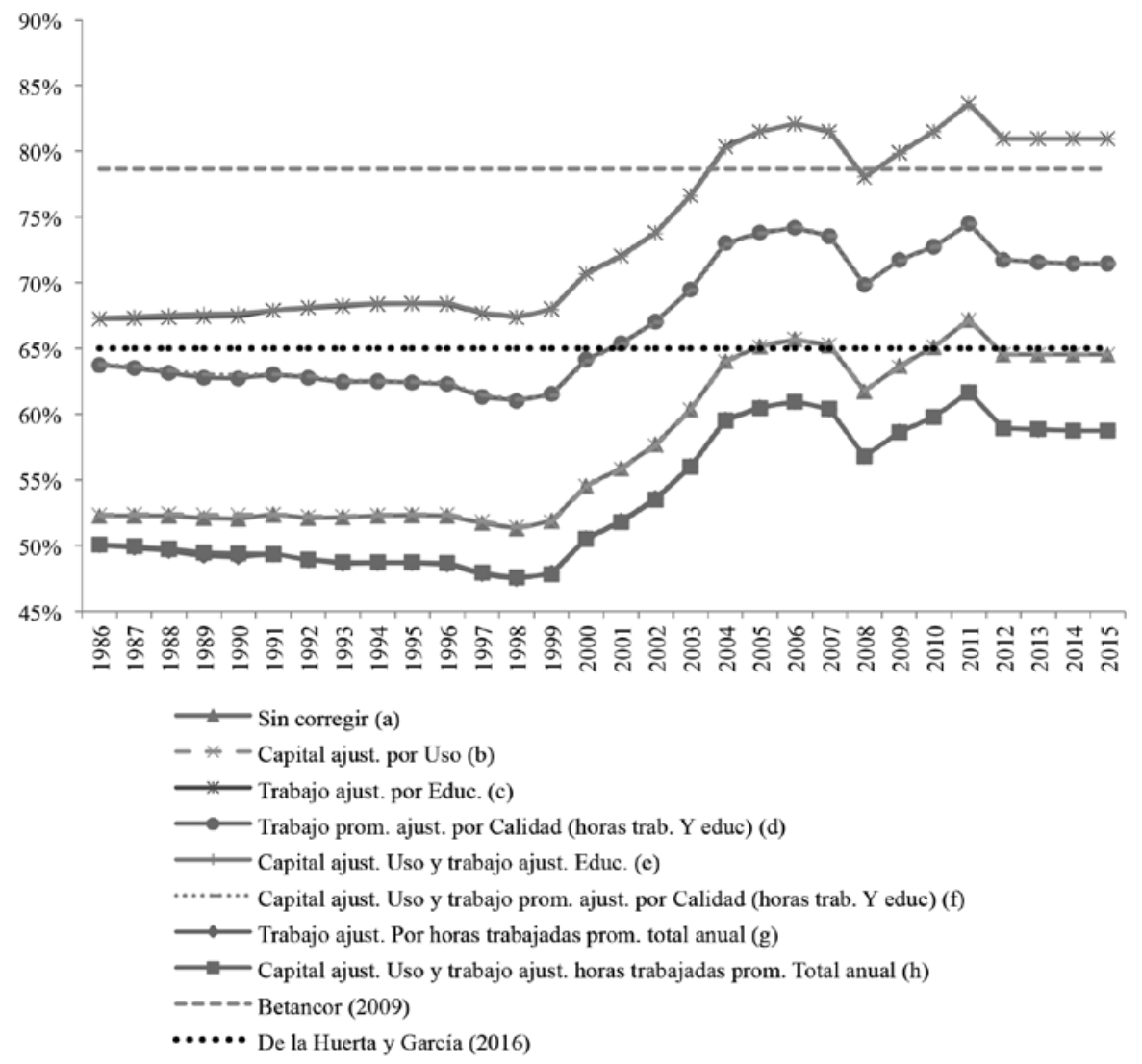

Nota 1: Las series a-h fueron obtenidas a partir de las estadísticas sectoriales de Cuentas Nacionales, ajustadas por los desvíos agregados entre Cuentas Nacionales y Hacienda, basado en la técnica de Vergara y Rivero (2006). Las distintas series corresponden a distintas medidas de trabajo y capital, ambas ajustadas y sin ajustar por calidad e intensidad de uso.

Nota 2: $a=\sin$ corregir; $b=$ capital ajustado por uso; $c=$ trabajo ajustado por educación; $d=$ trabajo ajustado por educación y horas trabajadas; $\mathrm{e}=$ capital ajustado por uso y trabajo ajustado por educación; $\mathrm{f}=$ capital ajustado por uso y trabajo ajustado por educación y horas trabajadas; $\mathrm{g}=$ trabajo ajustado por horas trabajadas; $\mathrm{h}=$ capital ajustado por uso y trabajo ajustado por horas trabajadas.

Nota 3: Se grafica a modo de comparación los parámetros fijos de participación utilizados por Betancor (2009) y De la Huerta y García (2016). 


\section{ESTIMACION DE LA PRODUCTIVIDAD TOTAL DE FACTORES DEL SECTOR CONSTRUCCION}

A continuación se calcula residualmente la productividad total de factores de la construcción, basado en la teoría de Solow previamente formalizada en la sección 2, y se analizan las principales fuentes del crecimiento del PIB sectorial. Para ello contamos con nueve series alternativas del stock de capital de la construcción (una medida sin ajustar y ocho series corregidas por la intensidad de uso del factor). A esto se suman cuatro series alternativas del factor trabajo (una sin ajustar y tres corregidas por calidad e intensidad de uso del mismo insumo) y un conjunto de 18 series opcionales de la tasa de contribución del trabajo en el PIB (parámetro $\alpha$ en la función de producción de Cobb-Douglas con el supuesto de rendimientos constantes a escala). La combinatoria de todas las medidas posibles de capital, mano de obra y tasa de participación del trabajo, arroja 648 medidas alternativas de la productividad total de factores del sector construcción.

La Figura 4 muestra el promedio de las 648 medidas alternativas de PTF obtenidas, con un intervalo del $95 \%$ de confianza. Esta representación permite comparar el promedio y la dispersión de las múltiples estimaciones de PTF obtenidas en el presente documento con las series generadas por Betancor (2009). Lo primero que podemos destacar es que, al considerar todas las medidas alternativas de PTF, existe un patrón de comportamiento similar entre ellas: la productividad sectorial no parece exhibir tendencia alguna en el período 1986-2009, coherente con la conclusión de Betancor (2009). El segundo resultado importante a destacar es que, independiente de la medición del factor utilizada o sus tasas de participación en el PIB, el nivel de la PTF sugiere una tendencia negativa a partir de 2010 hasta 2015. Si consideramos el ciclo expansivo que experimentó la actividad de la construcción durante el período 2011-2012 (7\% de crecimiento anual promedio), la reciente tendencia negativa de la PTF pone en evidencia el escaso protagonismo de la productividad en la dinámica del PIB de la construcción. Es decir, el crecimiento del sector es mayormente explicado por la acumulación de los factores respecto del uso eficiente de estos en el proceso constructivo.

De la Figura 4 se desprende además que las medidas selectivas de productividad calculadas por Betancor (2009) no solo se encuentran dentro del rango de estimaciones posibles, sino que sus patrones de comportamiento están altamente correlacionados con el promedio de las estimaciones alternativas de $\mathrm{PTF}^{15}$. Ello avala la robustez de las estimaciones anteriores de productividad a distintas y más recientes medidas de los factores y parámetros de contribución del trabajo en el PIB sectorial.

15 La correlación más alta $(0,7)$ ocurre entre la media de nuestras estimaciones y la PTF (con capital y trabajo sin ajustar) calculada por Betancor (2009). 


\section{FIGURA 4}

\section{ESTIMACION DE LA PRODUCTIVIDAD TOTAL DE FACTORES PARA \\ EL SECTOR CONSTRUCCION 1986-2015, INTERVALO AL 95\% \\ DE CONFIANZA VS. ESTIMACIONES BETANCOR (2009)}

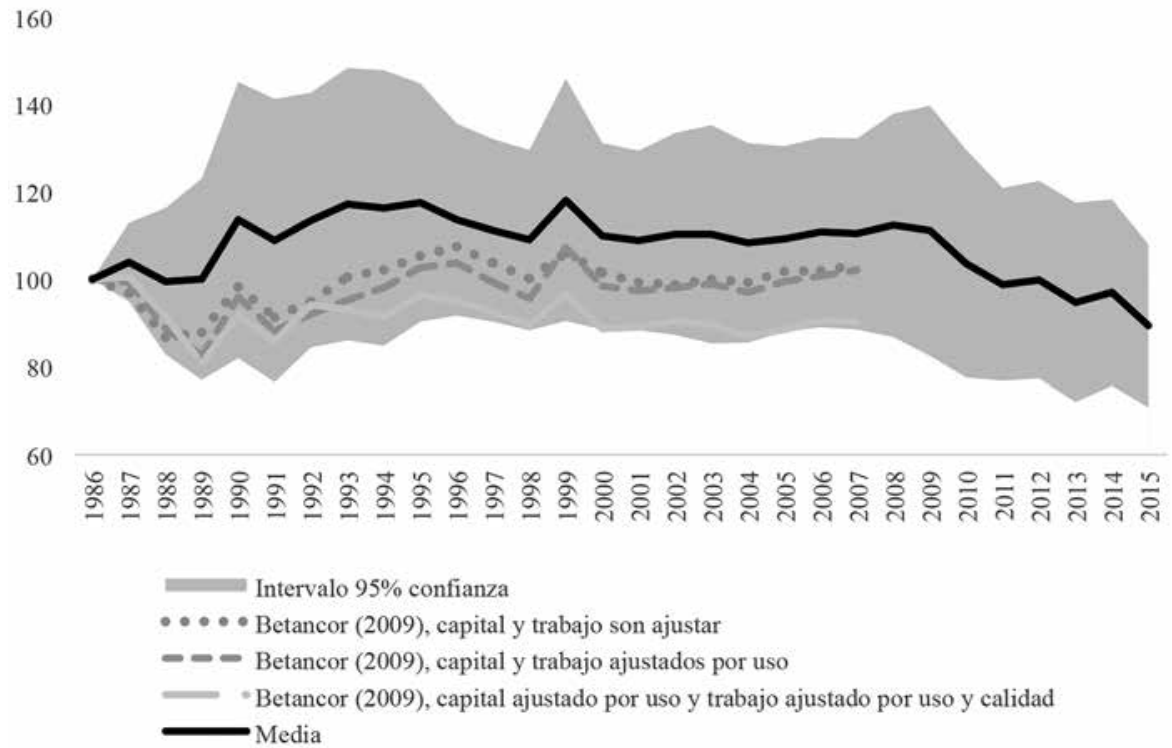

Determinado el período de análisis (1986-2015) y los supuestos del modelo neoclásico discutidos en la Sección 2, se tiene que el crecimiento observado en el sector construcción puede ser mayormente explicado por la acumulación de los factores primarios de producción (capital y trabajo) versus la eficiencia con que las empresas del sector utilizan sus insumos. Esto último podría sustentar, en parte, el alto grado de sensibilidad de la actividad de la construcción al ciclo económico. Por ejemplo, una recesión económica implicaría una brusca desacumulación de factores (aumento de la capacidad instalada ociosa por el menor uso del capital y mayores despidos de trabajadores), y por tanto, en un escenario de escasa productividad, no sería posible amortiguar la caída del producto agregado del sector. En consecuencia, el ciclo del PIB sectorial amplifica el ciclo del PIB total de la economía.

\subsection{La contribución de la productividad al crecimiento}

En este apartado se intenta responder la pregunta ¿En qué medida ha contribuido la PTF de la construcción al crecimiento del PIB del mismo sector? Para lograr una respuesta cuantitativa, basado en el método tradicional de Solow, se descompone el 
PIB de la construcción en sus principales fuentes del crecimiento: capital, trabajo y la productividad. A continuación, la Tabla 2 muestra la desagregación del crecimiento del PIB de la construcción en las contribuciones de los factores primarios de producción y el aporte de la productividad sectorial (PTF). Para ello se desagregó el período anual 1986-2015 en cinco subperíodos, con el propósito de identificar algún grado de heterogeneidad en el aporte de la PTF al crecimiento de la actividad sectorial.

Los resultados de la Tabla 2 también incluyen el aporte de la PTF sectorial diferenciando entre (i) las medidas tradicionales de los factores (sin ajustar por calidad e intensidad de uso del factor), y las medidas alternativas de capital y trabajo; (ii) ocupados de la construcción ajustados por horas trabajadas y años de educación; (iii) capital corregido por la intensidad de uso, utilizado como instrumento de ajuste las desviaciones a la tendencia de la fabricación de cemento; y (iv) capital ajustado por las desviaciones de los despachos industriales como instrumento alternativo de ajuste por intensidad de uso del factor.

Así, la primera columna de datos de la Tabla 2 corresponde al crecimiento anual del PIB de la construcción para cada subperíodo (en quinquenios) y el conjunto total de observaciones (1986-2015). La segunda y tercera columna de datos exhiben, respectivamente, las contribuciones tanto del capital como del trabajo al crecimiento del PIB sectorial. Por último, la columna de la PTF expone la participación porcentual de la productividad total de factores en el crecimiento del sector.

En términos cuantitativos, los resultados (i y ii) de la Tabla 2, basados en la medida tradicional del capital para el período completo 1986-2015, arrojan una contribución del stock del capital en torno a un punto porcentual de una tasa promedio de variación anual de 5,11\% del PIB de la construcción. Es decir, el acervo del capital explica cerca de un quinto del crecimiento del PIB sectorial durante el período de análisis. Este resultado es similar al obtenido a base de la medida de capital ajustado por su intensidad de uso (iii), en donde los ajustes provienen de las desviaciones a la tendencia cíclica de la fabricación de cemento. Por otra parte, cuando el stock de capital es ajustado por los desvíos de los despachos físicos industriales (medida alternativa de ajuste por el grado de utilización del factor en el proceso productivo), el aporte del capital disminuye marginalmente hasta 0,97 puntos porcentuales. En suma, el capital invertido en obras de edificación y obras civiles explica en torno al $20 \%$ del crecimiento anual de largo plazo del PIB de la construcción.

Por su parte, el factor trabajo, medido como el número de ocupados en el sector (i), explica 4,79 puntos porcentuales de los $5,11 \%$ de crecimiento promedio anual de la construcción durante 1986-2015; lo que significa que la mano de obra explica prácticamente la totalidad de los 5,11 puntos que creció el PIB sectorial durante el período bajo análisis. No obstante, esta medida aumenta hasta 4,9 puntos porcentuales cuando el trabajo es corregido por su calidad e intensidad de uso. En este caso, los ajustes son aproximados por los años de educación y las horas trabajadas, así como lo muestran los resultados ii, iii y iv de la Tabla 2. Con todo, el insumo trabajo utilizado en obras de edificación y obras civiles explica casi la totalidad del crecimiento anual 


\section{TABLA 2}

\section{FUENTES DEL CRECIMIENTO DEL PIB DE LA CONSTRUCCION EN CHILE, VARIOS SUBPERIODOS DE 1986-2015}

\begin{tabular}{|c|c|c|c|c|}
\hline Períodos & $\begin{array}{l}\text { Crecimiento } \\
\text { PIB (\%) }\end{array}$ & $\begin{array}{c}\text { Contribución } \\
\text { Capital (\%) }\end{array}$ & $\begin{array}{l}\text { Contribución } \\
\text { Trabajo (\%) }\end{array}$ & $\begin{array}{c}\text { Contribución } \\
\text { PTF (\%) }\end{array}$ \\
\hline \multicolumn{5}{|l|}{ (i) Capital y trabajo } \\
\hline 1986-1991 & 7,47 & $-1,19$ & 10,89 & $-2,23$ \\
\hline $1992-1997$ & 10,14 & 2,50 & 5,34 & 2,30 \\
\hline $1998-2003$ & $-0,37$ & $-0,18$ & 0,59 & $-0,79$ \\
\hline 2004-2009 & 4,61 & 1,43 & 2,61 & 0,57 \\
\hline 2010-2015 & 4,09 & 2,19 & 4,27 & $-2,37$ \\
\hline $1986-2015$ & 5,11 & 1,02 & 4,53 & $-0,44$ \\
\hline \multicolumn{5}{|c|}{ (ii) Capital y trabajo ajustado por horas y educación } \\
\hline 1986-1991 & 7,47 & $-1,19$ & 11,44 & $-2,78$ \\
\hline $1992-1997$ & 10,14 & 2,50 & 5,99 & 1,65 \\
\hline $1998-2003$ & $-0,37$ & $-0,18$ & 0,86 & $-1,05$ \\
\hline 2004-2009 & 4,61 & 1,43 & 3,00 & 0,17 \\
\hline 2010-2015 & 4,09 & 2,19 & 4,28 & $-2,38$ \\
\hline $1986-2015$ & 5,11 & 1,02 & 4,90 & $-0,81$ \\
\hline \multicolumn{5}{|c|}{ (iii) Capital ajustado por cemento y trabajo ajustado por horas y educación } \\
\hline 1986-1991 & 7,47 & $-1,21$ & 11,44 & $-2,76$ \\
\hline $1992-1997$ & 10,14 & 2,42 & 5,99 & 1,73 \\
\hline $1998-2003$ & $-0,37$ & $-0,04$ & 0,86 & $-1,20$ \\
\hline 2004-2009 & 4,61 & 1,32 & 3,00 & 0,29 \\
\hline 2010-2015 & 4,09 & 2,27 & 4,28 & $-2,46$ \\
\hline $1986-2015$ & 5,11 & 1,03 & 4,90 & $-0,82$ \\
\hline \multicolumn{5}{|c|}{ (iv) Capital ajustado por despachos industriales y trabajo ajustado por horas y educación } \\
\hline 1986-1991 & 7,47 & $-1,25$ & 11,44 & $-2,72$ \\
\hline $1992-1997$ & 10,14 & 2,45 & 5,99 & 1,69 \\
\hline $1998-2003$ & $-0,37$ & $-0,06$ & 0,86 & $-1,18$ \\
\hline 2004-2009 & 4,61 & 1,22 & 3,00 & 0,39 \\
\hline 2010-2015 & 4,09 & 2,09 & 4,28 & $-2,29$ \\
\hline $1986-2015$ & 5,11 & 0,97 & 4,90 & $-0,75$ \\
\hline
\end{tabular}

Nota: Se utilizó el filtro de Kalman como procedimiento de ajuste tendencial de las variables. El parámetro de participación del factor trabajo está basado en el estudio de Betancor (2009). 
de largo plazo del PIB de la construcción. Ello es coherente con el hecho de que la actividad sectorial es intensiva en el uso de la mano de obra, siendo uno de los sectores de la economía que aporta significativamente al empleo total del país ${ }^{16}$.

Por otra parte, la productividad total de factores (PTF), independiente de la medida de los factores primarios utilizada, ha contribuido negativamente al crecimiento del PIB de la construcción durante todo el período de análisis (1986-2015). Es decir, el uso ineficiente de los insumos por parte de las empresas del sector le ha restado crecimiento al valor agregado de la construcción (entre 0,4 y 0,8 puntos porcentuales). Al respecto, es posible señalar que este resultado está afectado por la fuerte caída de la productividad durante los quinquenios que incluyen la crisis asiática y la crisis subprime, y donde la heterogeneidad en los resultados es evidente cuando observamos la descomposición del PIB de la construcción y sus fuentes de crecimiento en subperíodos de cinco años. Por ejemplo, la contribución de la PTF al crecimiento del PIB de la construcción resultó positiva en el quinquenio 1992-1997; período que coincide con un sustancial crecimiento del sector $\left(10,1 \%\right.$ anual) y de la economía en general ${ }^{17}$. En esta ocasión, considerando las medidas tradicionales del capital y trabajo (i), la productividad explicó cerca del $22 \%$ del crecimiento anual del PIB de la construcción. Mientras que, al ajustar los factores por calidad e intensidad de uso (ii, iii y iv), se tiene que la PTF explicó algo más que $16 \%$ del alza promedio del PIB sectorial.

Con todo, es admisible destacar que estos resultados no están exentos de algunas limitaciones, como la forma funcional y los supuestos de rendimientos a escala para modelar el PIB de la construcción, los instrumentos y técnicas de ajuste de los factores por su calidad e intensidad de uso en el proceso productivo. La calidad de estos supuestos se refleja marcadamente en el cálculo residual de la PTF, y las estimaciones de productividad y su contribución al crecimiento constituyen entonces solo una aproximación de la realidad sectorial. Sin embargo, pese a esta y otras limitantes, este procedimiento de cálculo de la PTF sigue siendo el principal referente en el mundo académico.

La Figura 5 resume gráficamente la descomposición del PIB de la construcción en las contribuciones de los factores de producción, basado en los parámetros de participación del trabajo de Betancor (2009) y De la Huerta y García (2016). Los gráficos (a) y (c) de la Figura 5 muestran diferencias poco disímiles en las contribuciones de la productividad al crecimiento del PIB sectorial a partir del quinquenio 19982003. En tanto, previo a dicho período, el cálculo basado en los parámetros de De la Huerta y García (2016) provee evidencia de un aporte prácticamente nulo de la PTF al crecimiento sectorial en el período 1986-1991, para luego en el quinquenio 1992-1997 resultar negativo.

16 El empleo de la construcción representa cerca del 9\% del agregado de ocupados en la economía, según los registros del INE.

17 El PIB agregado de la economía creció más de 7\% anual en el quinquenio 1992-1997. 


\section{FIGURA 5}

\section{FUENTES DE CRECIMIENTO DEL PIB DE LA CONSTRUCCION, VARIOS SUBPERIODOS Y MEDIDAS DE FACTORES}

(a) Capital ajustado por despachos físicos industriales y trabajo ajustado por horas y educación.

Utilizando los parámetros de Betancor (2009)

$15 \%$
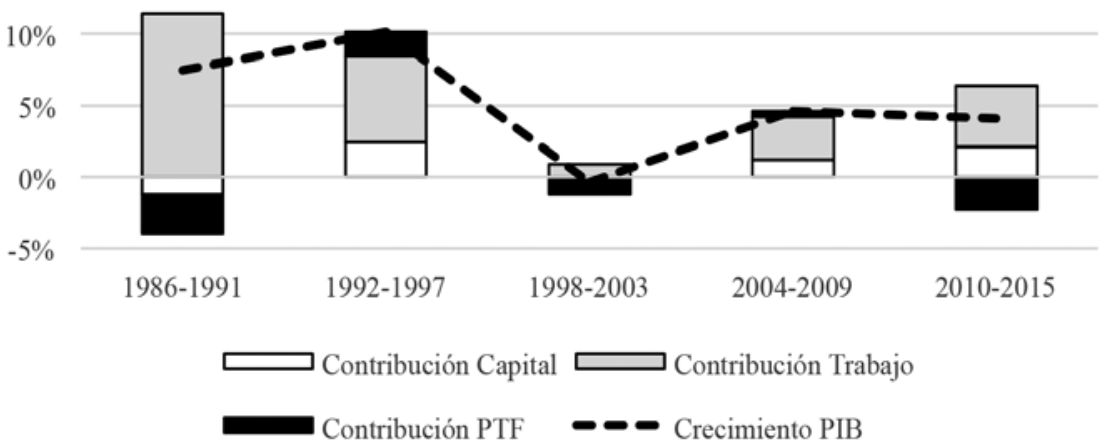

(b) Período 1986-2015 con cuatro medidas alternativas de factores básicos de producción.

Utilizando los parámetros de Betancor (2009)

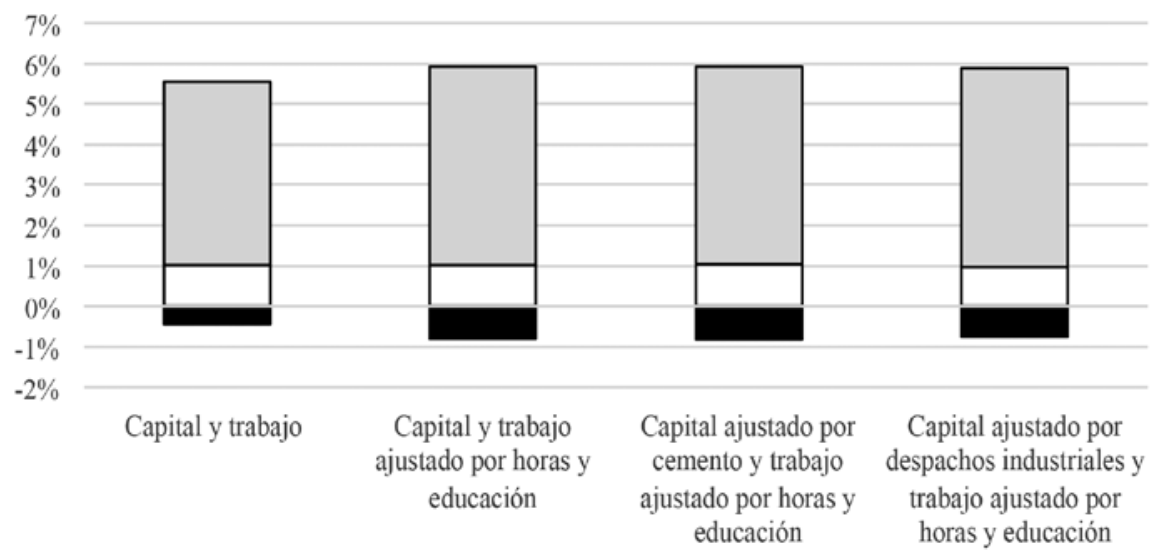

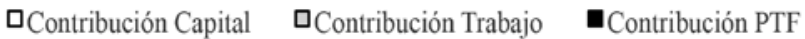


(c) Capital ajustado por despachos físicos industriales y trabajo ajustado por horas y edución.

Utilizando los parámetros de De la Huerta y García (2016)

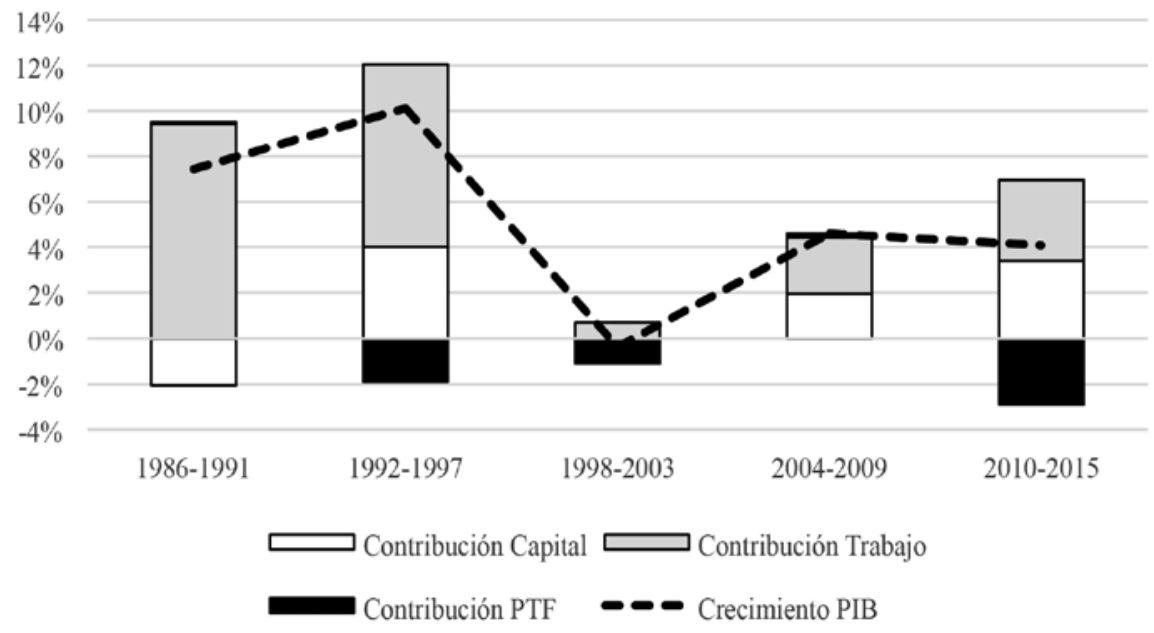

(d) Período 1986-2015 con cuatro medidas alternativas de factores básicos de producción. Utilizando los parámetros de De la Huerta y García (2016)

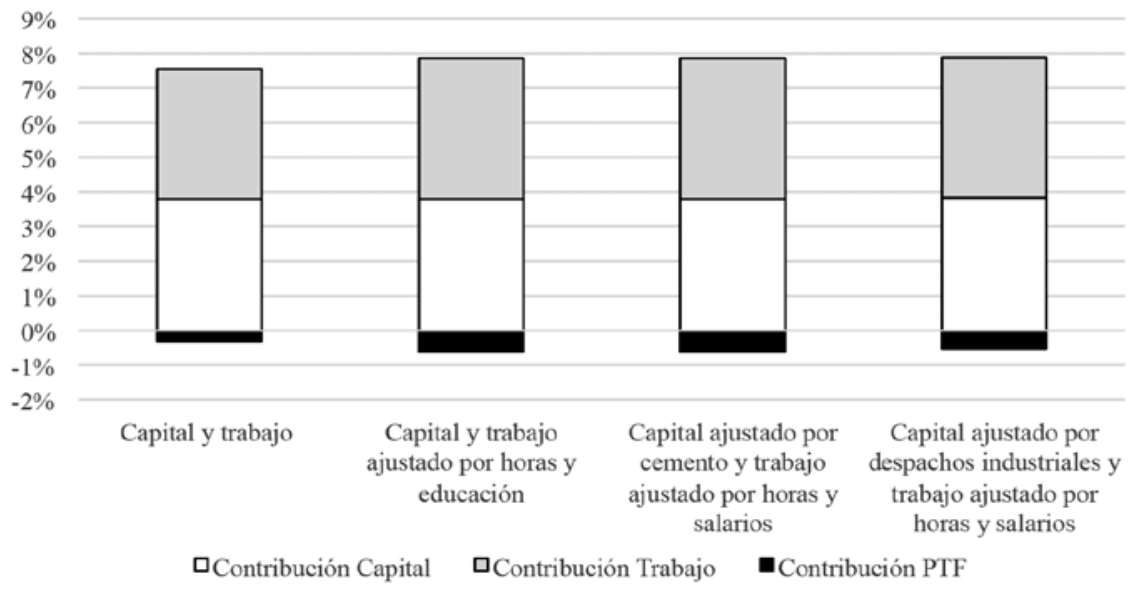


Por el contrario, a partir de los resultados basados en Betancor (2009), se observa un aporte negativo de la productividad al crecimiento durante 1986-1991. No obstante, la contribución de la PTF al crecimiento del PIB de la construcción resulta positiva en los años 1992-1997. Ello, entre otros factores, puede deberse a la menor calidad de los datos de aquellos años o la dificultad por replicar una evolución altamente volátil del PIB de la construcción.

En contraste, los gráficos (b) y (d) muestran que la contribución de la productividad al crecimiento de la actividad de la construcción es negativa para el período completo 1986-2015, independiente de la medida de ajuste que se aplique a los factores de producción y de la fuente de sus tasas de participación en el PIB. Esto implicaría que el crecimiento de largo plazo experimentado por el sector construcción presenta un escaso protagonismo de mejoras de su productividad, siendo prácticamente dominado por la mera acumulación de los factores primarios de producción (trabajo y capital).

Finalmente, es interesante notar que, a nivel agregado, existe un comportamiento similar al observado a nivel sectorial en este estudio, si se compara el aporte de la productividad al crecimiento del PIB en distintos períodos del ciclo económico. Por ejemplo, Beyer y Vergara (2002) y Fuentes, Larraín y Schmidt-Hebbel, K. (2004) encuentran que la contribución de la productividad al crecimiento del PIB es menor en la década de los 80 respecto de lo observado en la primera mitad de los años $90^{18}$. No obstante, la contribución de la productividad exhibe una importante caída en los años de la crisis asiática (1998-2003), lo que les permite concluir a los autores que la PTF a un nivel agregado presenta un carácter fuertemente procíclico, patrón que observamos en los resultados obtenidos para la contribución de la productividad al crecimiento sectorial (Figura 5 (a) y (c)). De este modo, el comportamiento de la productividad de la construcción posee características procíclicas -similar a la dinámica exhibida por la productividad agregada de la economía en las distintas etapas del ciclo económico-.

\section{CONCLUSIONES}

- La productividad total de factores del sector construcción se ha mantenido prácticamente plana en el período 1986-2009, consistente con las conclusiones que se desprenden del trabajo de Betancor (2009). No obstante, a partir del año 2010 hasta 2015 la medida de productividad exhibe una tendencia decreciente. Si consideramos el ciclo expansivo que experimentó la actividad de la construcción

18 Beyer y Vergara (2002) estiman que el aporte de la productividad al crecimiento del PIB sería de 2,3\% entre 1986 y 1990, mientras que este alcanzaría 3,7\% entre 1991 y 1995, para luego caer a $-0,6 \%$ entre 1998 y 2001. Por otro lado, al corregir capital y trabajo por horas y salario, Fuentes, Larraín y Schmidt-Hebbel (2004) estiman que entre 1974-1989 el aporte de la productividad alcanza $-0,89 \%$, alzándose a 4,02\% entre 1990-1997 y volviendo a caer a 0,25\% entre 1998-2003. 
durante el período 2011-2012 (7\% de crecimiento anual promedio), la reciente tendencia negativa de la productividad pone en evidencia su escaso protagonismo en la dinámica del PIB de la construcción. Es decir, el crecimiento del sector es mayormente explicado por la mera acumulación de los factores y no por el uso eficiente de los recursos en el proceso constructivo.

- En el período 1986-2015 los resultados revelan una contribución negativa de la productividad al crecimiento del PIB de la construcción. Es decir, el uso ineficiente de los insumos por parte de las empresas del sector le ha restado crecimiento al valor agregado de la construcción (entre 0,4 y 0,8 puntos porcentuales). Al respecto, es sugestivo señalar que este resultado es arrastrado por la fuerte caída de la productividad durante los períodos que incluyen la crisis asiática y la crisis subprime.

- La heterogeneidad en los resultados es evidente cuando observamos la descomposición del PIB de la construcción y sus fuentes de crecimiento en subperíodos de cinco años. Particularmente, la contribución de la productividad al crecimiento del PIB de la construcción resultó positiva en el quinquenio 1992-1997; período que coincide con un sustancial crecimiento del sector (10,1\% anual), y donde una mayor eficiencia en el uso de los recursos explicaría entre $16 \%$ y $22 \%$ del alza promedio del PIB sectorial -según se corrija o no los factores productivos por su calidad e intensidad de uso-.

- Sin embargo, al consolidar todo el período de análisis (1986-2015), el crecimiento de largo plazo experimentado por el sector construcción presenta un escaso protagonismo de mejoras de su productividad, siendo prácticamente dominado por la mera acumulación de los factores primarios de producción (trabajo y capital). Esta situación es alarmante para el futuro desempeño del sector, ya que su escasa o nula productividad lo hace especialmente vulnerable a los altos y bajos del ciclo económico.

\section{BIBLIOGRAFIA}

ASTORGA, R. (2011). "Causas del menor ritmo de crecimiento económico en Chile: una perspectiva sectorial", Informe de tesis para optar al grado de Magíster en Economía. Universidad de Chile.

BERGOEING, R., KEHOE, P., KEHOE, T., y SOTO, R. (2002). "Policy-Driven Productivity in Chile and Mexico in the 1980s and 1990s", American Economic Review, Papers and Proceedings 92 (2): 16-21.

BETANCOR, A. (2009). "Productividad del Sector Construcción", Documentos de Trabajo No 40, Cámara Chilena de la Construcción.

BEYER, H., y VERGARA, R. (2002). "Productivity and Economic Growth: The Case of Chile", en N. Loayza y R. Soto (eds.). Economic Growth: Sources, Trends and Cycles, Banco Central de Chile.

CONTRERAS, G., y GARCIA, P. (2002). "Estimating Gaps and Trends for the Chilean Economy", Revista de Economía Chilena 5 (2).

COEYMANS, J. E. (1999). "Determinantes de la Productividad en Chile", Cuadernos de Economía 107: 597-637.

CHUMACERO, R. y FUENTES, R. (2006). “Chilean Growth Dynamics”, Economic Modelling, 23 (2): 197-214. 
DE GREGORIO, J. (1997). "Crecimiento Potencial en Chile: Una Síntesis”, en Morandé y Vergara (eds.). Análisis Empírico del Crecimiento Económico en Chile, Centro de Estudios Públicos, ILADES/ Georgetown.

DE GREGORIO, J. y LEE, J. W. (1999). "Economic Growth in Latin America: Sources and Prospects", Documentos de Trabajo $\mathrm{N}^{\circ}$ 66, Centro de Economía Aplicada, Universidad de Chile.

DE LA HUERTA, C., y GARCIA, C. (2016). "Commodity Prices, Growth and Productivity: a Sectoral View”, Documentos de Trabajo No 777, Banco Central de Chile.

FUENTES, R.; LARRAIN, M.; SCHMIDT-HEBBEL, K. (2004). "Fuentes del Crecimiento y Comportamiento de la Productividad Total de Factores en Chile", Documentos de Trabajo № 287, Banco Central de Chile.

GALLEGO, F., y LOAYZA, N. (2002). "The Golden Period for Growth in Chile. Explanations and Forecasts", en N. Loayza y R. Soto (eds.). Economic Growth: Sources, Trends and Cycles, Banco Central de Chile.

MAGENDZO, I., y VILLENA, M. (2012). "Evolución de la Productividad Total de Factores en Chile", Informe Técnico de la Universidad Adolfo Ibáñez. Marzo 2012.

SOLIMANO, A., y SOTO, R. (2003). "Latin American Economic Growth in the Late 20th Century: Evidence and Interpretation". Preparado para el Seminario "Latin America Growth: Why so Slow?".

ROJAS, P.; LOPEZ, E., y JIMENEZ, S. (1997). "Determinantes del crecimiento y estimación del producto potencial en Chile: El rol del comercio internacional", en Morandé y Vergara (eds.). Análisis Empírico del Crecimiento Económico en Chile, Centro de Estudios Públicos, ILADES/Georgetown.

ROLDOS, J. (1997). "El crecimiento del producto potencial en mercados emergentes: El caso de Chile", en Morandé y Vergara (eds.). Análisis Empírico del Crecimiento Económico en Chile, Centro de Estudios Públicos, ILADES/Georgetown.

SOLOW, R. (1957). "Technical Change and the Aggregate Production Function", Review of Economics and Statistics 39: 312-320.

VERGARA, R. y RIVERO, R. (2006). "Productividad Sectorial en Chile: 1986-2001", Cuadernos de Economía, Vol. 43, pp. 143-168. Mayo 2006. 